\title{
EXACT SOLUTIONS FOR AXIAL STATIC ANALYSIS OF NANORODS USING WEIGHTED RESIDUALS
}

\author{
Mustafa Özgür YAYLI*', Uğur KAFKAS, Büşra UZUN
}

Bursa Uludag University, Engineering Faculty, Department of Civil Engineering, Bursa, Turkey

\begin{tabular}{l}
\hline Keywords \\
\hline Axial Static, \\
Nanorod, \\
Weighted Residual Methods, \\
Displacement Function, \\
Exact Solution.
\end{tabular}

\begin{abstract}
In the present work, axial static analysis of nanorods under triangular loading is presented via Eringen's nonlocal differential model. Three weighted residual methods (Subdomain, Galerkin and Least squares methods) are used to obtain the exact static deflection. These methods require that the integral of the error with different assumptions over the domain be set to zero. The number of equations have to be equal to unknown terms. A cubic displacement function has been chosen for three weighted residual methods. Subdomain, Galerkin and Least squares methods yield identical solution as the exact solution. The plots of the solution are shown for different number of unknown coefficients.
\end{abstract}

\section{AĞIRLIKLI ARTIKLAR KULLANILARAK NANOÇUBUKLARIN EKSENEL STATİK ANALİZi İÇÍN KESİN ÇÖZÜMLER}

\begin{tabular}{l}
\hline Anahtar Kelimeler \\
\hline Eksenel Statik, \\
Nanoçubuk, \\
Ağırlıklı Artık Yöntemleri, \\
Yer Değiștirme Fonksiyonu, \\
Gerçek Çözüm.
\end{tabular}

Gerçek Cözüm.

\begin{abstract}
Öz
Bu çalışmada, Eringen'in yerel olmayan diferansiyel modeli kullanılarak; üçhen yayılı yüklenmiş nano çubukların eksenel statik analizi verilmiștir. Üç ağırlıklı artık tabanlı yöntem (Subdomain, Galerkin ve Least squares yöntemleri) gerçek statik deplasmanı elde etmek için kullanılmıştır. Bu yöntemler bölgenin tamamında integral hatalarını minimize etme varsayımına dayanmaktadır. Sistem denklemleri çözümü aranan bilinmeyenler ile aynı sayıda olmadır. Bu yüzden üç ağırlıklı artık yöntemi için de kübik polinomlar statik deplasmanı göstermek üzere seçilmiştir. Subdomain, Galerkin and Least squares yöntemleriyle gerçek çözümler ile aynı polinomlar olarak elde edilmiştir. Değişik sayıda bilinmeyen içeren sabitler ile grafikler çizdirilerek çözümler gösterilmiştir.
\end{abstract}

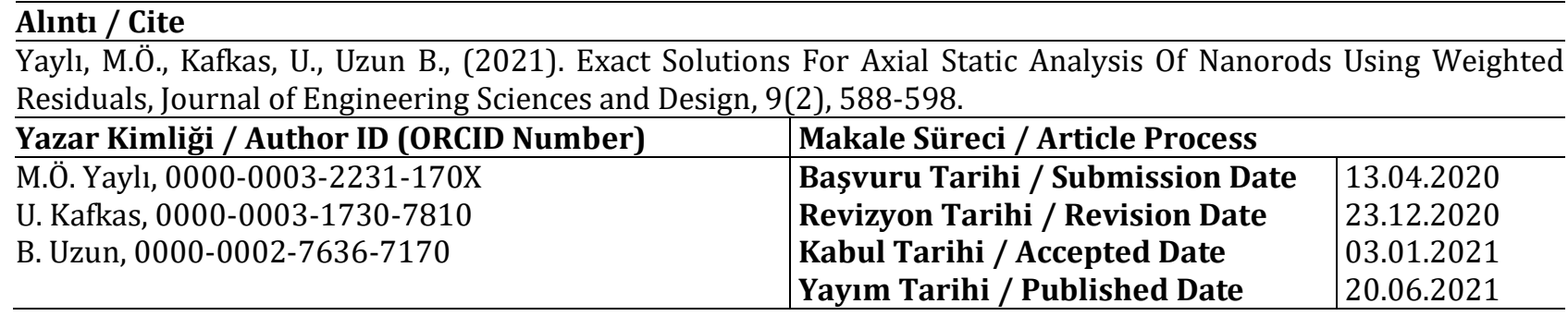

\section{Introduction}

The analytical and computer-based modeling of the dynamical or static behavior of nanobeams (carbon nanotubes) have been come the focal points of researches in computational material physics over the recent years. Moreover, many researhers have studied a structural mechanics and continuum approaches for more efficient and practical modeling. For this purpose, beam, plate and rod theories have been employed by several researchers. In the literature, there have been several number of researches, both theoretical and atomistic, of the mechanical properties of nanobeams. The mechanical analysis of nanosized beams has been implemented using several types of classic elasticity theories, namely based on Euler Bernoulli or Timoshenko beam theories, frame models and plate-shell theories. However, these classical mechanics theories do not account for different phenomena that appear at the small size, such as molecular interactions of atoms and their effects. To overcome these shortcomings, different methods of non-classical elasticity models based on nonlocal elasticity theories have been

\footnotetext{
* İlgili yazar / Corresponding author: ozguryayli@uludag.edu.tr, +90-224-294-19-61
} 
constructed and utilized to nanobeams in recent years. The classical continuum based models are not able to predict higher order effects due to the absence of the nonlocal parameter. To overcome these shortcomings, different nonlocal stress or strain gradient elasticity theories (modified couple stress theory, nonlocal elasticity theory, couple stress theory, strain gradient elasticity theory etc...) have been used to solve these non-classical problems.

Mechanical behavior of rods and nono rods is one of the most frequently studied topics (Arda and Aydogdu 2014; Arda and Aydogdu 2016; Aydogdu and Arda 2016; Arda and Aydogdu 2017; Arda and Aydogdu 2018; Akbaş 2019; Akgöz 2019; Uzun and Yaylı 2020a, Uzun et al. 2020a). More appropriate elasticity theories rather than local or classical shell, plate and beam theories are needed in investigating the size effect in nano-structures. So far several higher order elasticity models have been proposed to understand the small size effect (Li, 2014; Li et al., 2015; Li et al., 2017; Civalek and Demir, 2016; Liu et al., 2017; Kounadis et al., 2006; Akgöz and Civalek, 2013; ; Akgöz and Civalek, 2015; Ansari et al., 2013; Thai et al. 2018; Ebrahimi et al. 2019; Jalaei and Civalek 2019; Arefi et al. 2020; Demir and Civalek 2013; Dastjerdi et al. 2020, Uzun and Yaylı 2020b, Uzun et al. 2020b, c). From the academic studies in the literature, the mechanical methods adopted to explore the dynamical and atomistic behavior of nanobeams can be divided in to three main categories: the atomistic approaches, higher order elasticity theories (gradient elasticity, nonlocal elasticity, modified couple stress theory. etc.) and the classical continuum mechanics approaches. Since the several classical models can not predict the static deflection or dynamical behaviour of nanobeams, different higher elasticity theories have been recently presented to predict the material properties of nanotubes (nanobeams, nanorods) in recent years (Ece and Aydoğdu, 2007; Şimșek, 2010; Aydoğdu, 2009; Reddy and Pang, 2008; Aydoğdu, 2009; Yoon et al., 2003; Wang and Liew, 2007; Yaylı, 2017; Yayl, 2016a; Yaylı et al., 2015; Yaylı, 2016b; Yaylı, 2014, Civalek et al. 2020, Uzun et al. 2020d, e).

In this work, with regard to the importance of analyzing the axial deflection of nanorods and their vast range of applications, the axial static analsis with linear loading conditions is investigated to predict the impact of small scale parameter on axial deflections of nanorods by wighted residuals. In this study therefore, based on the Eringen's nonlocal elasticity theory, the static problem of axial deformations of a nanorod is tackled and investigated with the weighted residuals methods (Galerkin, subdomain, least squares methods). Moreover, the effect of the number of terms used in the polynomial and the nonlocal parameter of axial deflection of the nanorod has been addressed. A number of graphical results for the axial deflections of a nanorod by using weighted results are presented.

\section{Nonlocal Elasticity Theory}

For homogenous-isotropic elastic solids, Eringen's nonlocal elasticity theory is defined by the following equations (Eringen and Edelen, 1972; Eringen, 1983):

$$
\begin{aligned}
& \sigma_{k l, l}+\rho\left(f_{l}-\frac{\partial^{2} u_{l}}{\partial t^{2}}\right)=0 \\
& \sigma_{k l}(x)=\int_{V} \alpha\left(\left|x-x^{\prime}\right|, X\right) T_{k l}\left(x^{\prime}\right) d V\left(x^{\prime}\right) \\
& T_{k l}\left(x^{\prime}\right)=\lambda \varepsilon_{m m}\left(x^{\prime}\right) \delta_{k l}+2 \mu \varepsilon_{k l}\left(x^{\prime}\right) \\
& \varepsilon_{k l}\left(x^{\prime}\right)=\frac{1}{2}\left(\frac{\partial u_{k}\left(x^{\prime}\right)}{\partial x_{l}^{\prime}}+\frac{\partial u_{l}\left(x^{\prime}\right)}{\partial x_{k}^{\prime}}\right)
\end{aligned}
$$

in which, $\sigma_{k l}$ is the nonlocal stress tensor, $\rho$ is the mass density of the body, $u_{l}$ is the displacement vector, $\alpha / x /$ can be displayed by a linear differential operator, $f_{l}$ is the applied force density, $\tau_{k l}\left(x^{\prime}\right)$ is the Cauchy stress tensor at any point $x^{\prime}, \varepsilon_{k l}\left(x^{\prime}\right)$ is the strain tensor, $\mu$ and $\lambda$ are Lame constants, $V$ is the volume occupied by the body, $t$ denotes the time $\alpha / x-x^{\prime} \mid$ is the distance form of Euclidean. The following relation can be used in nonlocal elasticity (Eringen and Edelen, 1972; Eringen, 1983):

$$
\Gamma \alpha\left(\left|x-x^{\prime}\right|\right)=\delta\left(\left|x-x^{\prime}\right|\right)
$$

where $\delta[\mathrm{x}]$ is the nonlocal distance. The following operator has been derived from equation (2):

$$
\Gamma \alpha_{k l}=T_{k l}
$$

In which $\alpha[\mathrm{x}]$ is the nonlocal kernel. Furthermore, following relation can be derived from equation (1): 


$$
T_{k l, l}+\Gamma\left(f_{l}-\rho \ddot{u}_{k}\right)=0
$$

the differential operator has been expressed as the following compact form:

$$
\Gamma=\left(1-\left(e_{0} a\right)^{2} \nabla^{2}\right)
$$

in which $e_{0} a$ is the nonlocal parameter, $\nabla^{2}$ is the Laplacian. Then constitutive equation in Eringen's nonlocal elasticity may be expressed in terms of nonlocal parameter

$$
\left[1-\left(e_{0} a\right)^{2} \nabla^{2}\right] \sigma_{k l}=T_{k l}
$$

\section{Governing Equation}

By using the relation in (9), the governing equation in terms of the axial deflection as follows (Aydoğdu, 2009):

$$
\begin{aligned}
& E A \frac{d^{2} u(x)}{d x^{2}}+q(x)-\mu \frac{d^{2} q(x)}{d x^{2}}=0 \\
& \left(e_{0} a\right)^{2}=\mu
\end{aligned}
$$

where $u(x)$ denotes the axial deflection, $E$ is the modulus of elasticity, $A$ is the cross sectional area. Eq. (10) is the governing differential equation for the static deflection of nanorod. Integrating the above equation with respect to $x$ :

$$
E A \frac{d u(x)}{d x}-\mu \frac{d q(x)}{d x}=\int_{0}^{x} q(x) d x
$$

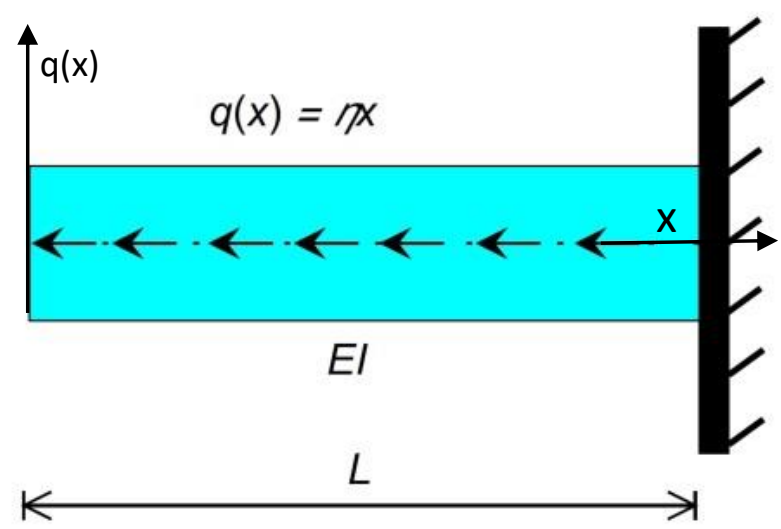

Figure 1. Axially loaded cantilever nanorod

Only one strict boundary condition was used in our study. When $\mathrm{x}=\mathrm{L}, \mathrm{u}=0$. For the other boundary condition, scientific debate continues that $d u / d x$ is zero when $x=0$. It is clear that the derivative is not necessarily zero, as the force is the boundary condition here. In this study, the cantilever nanorod in Figure 1 carries a triangular axial load:

$$
q(x)=\eta x
$$

where $\eta$ is a coefficient which represents the slope of triangular line load.

\section{Weighted Residuals Methods}

\subsection{Least squares method}

The method of least squares requires the integral over the domain residual function have to be minimized with respect to unknown terms: 


$$
\frac{\partial}{\partial c_{i}}\left(\int_{0}^{L} R^{2} d x\right)=0, \quad i=1,2, \ldots, N
$$

The above equation can be written as follows;

$$
\left(\int_{0}^{L} R \frac{\partial R}{\partial c_{i}} d x\right)=0
$$

Taking the slope $\eta=10$, the following relations can be written by using the Eq. 15 :

$$
\begin{aligned}
& \int_{0}^{L} A E\left(A E\left(3 c_{3}(x-L)^{2}+2 c_{2}(x-L)+c_{1}\right)\right. \\
& \int_{0}^{L} A E 2(x-L)\left(A E\left(3 x_{3}\right) d x=0\right. \\
& \left.\left.+c_{1}\right)-10 \mu+5 x^{2}\right) d x=0 \\
& \left.\int_{0}^{L} A E 3(x-L)^{2}+2 c_{2}(x-L)-10 \mu+5 x^{2}\right) d x=0
\end{aligned}
$$

Taking the definite integrals; following systems of linear equations are obtained:

$$
\begin{aligned}
& A^{2} c_{3} E^{2} L^{3}-A^{2} c_{2} E^{2} L^{2}+A^{2} c_{1} E^{2} L \\
& +\frac{5}{3} A E L^{3}-10 A E \mu L=0 \\
& -\frac{3}{2} A^{2} c_{3} E^{2} L^{4}+\frac{4}{3} A^{2} c_{2} E^{2} L^{3}-A^{2} c_{1} E^{2} L^{2} \\
& -\frac{5}{6} A E L^{4}+10 A E \mu L^{2}=0 \\
& \frac{9}{5} A^{2} c_{3} E^{2} L^{5}-\frac{3}{2} A^{2} c_{2} E^{2} L^{4}+A^{2} c_{1} E^{2} L^{3} \\
& +\frac{1}{2} A E L^{5}-10 A E \mu L^{3}=0
\end{aligned}
$$

Solving the above equations, we obtain the unknown coefficients as follows:

$$
\begin{aligned}
& c_{1}=-\frac{5\left(L^{2}-2 \mu\right)}{A E} \\
& c_{2}=-\frac{5 L}{A E} \\
& c_{3}=-\frac{5}{3 A E}
\end{aligned}
$$

Substituting the symbolic values for the coefficients given in deflection function:

$$
\bar{U}_{L S M}=\frac{5\left(L^{3}-6 \mu L-x^{3}+6 \mu x\right)}{3 A E}
$$

\subsection{Subdomain Method}

Subdomain method require the integral over the some selected domains residual function be set zero:

$$
\int_{0}^{L / 3} R d x=\int_{0}^{L / 3} E A\left(3 c_{3}(x-L)^{2}+2 c_{2}(x-L)\right.
$$




$$
\begin{aligned}
& \int_{L / 3}^{2 L / 3} R d x=\int_{L / 3}^{2 L / 3} E A\left(3 c_{3}(x-L)^{2}+2 c_{2}(x-L)-10 \mu+5 x^{2} d x=0\right. \\
& \left.\int_{2 L / 3}^{L} R d x=\int_{2 L / 3}^{L}+c_{1}\right)-10 \mu+5 x^{2} d x=0
\end{aligned}
$$

Calculating the above integrals gives the following systems of equations;

$$
\begin{aligned}
& \frac{19}{27} A c_{3} E L^{3}-\frac{5}{9} A c_{2} E L^{2}+\frac{1}{3} A c_{1} E L \\
& +\frac{5 L^{3}}{81}-\frac{10 \mu L}{3}=0 \\
& \frac{7}{27} A c_{3} E L^{3}-\frac{1}{3} A c_{2} E L^{2}+\frac{1}{3} A c_{1} E L \\
& +\frac{35 L^{3}}{81}-\frac{10 \mu L}{3}=0 \\
& \frac{1}{27} A c_{3} E L^{3}-\frac{1}{9} A c_{2} E L^{2}+\frac{1}{3} A c_{1} E L \\
& +\frac{95 L^{3}}{81}-\frac{10 \mu L}{3}=0
\end{aligned}
$$

By combining these three equations as a system, these can be written in matrix form as follows:

$$
\left(\begin{array}{lll}
a_{11} & a_{12} & a_{13} \\
a_{21} & a_{22} & a_{23} \\
a_{31} & a_{32} & a_{33}
\end{array}\right)\left(\begin{array}{l}
c_{1} \\
c_{2} \\
c_{3}
\end{array}\right)=\left(\begin{array}{c}
-\frac{5 L^{3}}{81}+\frac{10 \mu L}{3} \\
-\frac{35 L^{3}}{81}+\frac{10 \mu L}{3} \\
-\frac{95 L^{3}}{81}+\frac{10 \mu L}{3}
\end{array}\right)
$$

We have obtained the unknown coefficient expressions as same as the Eqs. 22, 23 and 24. Substituting these coefficients in deflection function then the same relation obtained from Eq. 25.

\subsection{Galerkin Method}

The method of Galerkin requires the residual function to be ortogonal to other weighting functions:

$$
\left(\int_{0}^{L} R W_{i} d x\right)=0, \quad i=1,2, \ldots, N
$$

In this study, the weighting function formulas are selected to be a part of deflection function. The title problem of this letter have three unknown coefficients $\left(c_{1}, c_{2}, c_{3}\right)$, therefore following three weighting functions are chosen;

$$
\begin{aligned}
& W_{1}=x-L \\
& W_{2}=(x-L)^{2} \\
& W_{3}=(x-L)^{3}
\end{aligned}
$$

Using the above approximation functions, the following relations are generated.

$$
\begin{aligned}
& \int_{0}^{L} A E\left(A E\left(3 c_{3}(x-L)^{2}+2 c_{2}(x-L)+c_{1}\right)\right. \\
& \int_{0}^{L} A E 2\left(x-L x^{2}\right)(x-L) d x=0 \\
& \left.\left.+c_{1}\right)-10 \mu+5 x^{2}\right)\left(x-L c_{3}(x-L)^{2}+2 c_{2}(x-L)\right.
\end{aligned}
$$




$$
\begin{aligned}
& \int_{0}^{L} A E 3(x-L)^{2}\left(A E\left(3 c_{3}(x-L)^{2}+2 c_{2}(x-L)-10 \mu+5 x^{2}\right)(x-L)^{3} d x=0\right.
\end{aligned}
$$

Computing the above integrals; the following systems equations are derived:

$$
\begin{aligned}
& -\frac{3}{4} A c_{3} E L^{4}+\frac{2}{3} A c_{2} E L^{3}-\frac{1}{2} A c_{1} E L^{2} \\
& -\frac{5 L^{4}}{12}+5 \mu L^{2}=0 \\
& \frac{3}{5} A c_{3} E L^{5}-\frac{1}{2} A c_{2} E L^{4}+\frac{1}{3} A c_{1} E L^{3} \\
& +\frac{L^{5}}{6}-\frac{10 \mu L^{3}}{3}=0 \\
& -\frac{1}{2} A c_{3} E L^{6}+\frac{2}{5} A c_{2} E L^{5}-\frac{1}{4} A c_{1} E L^{4} \\
& -\frac{L^{6}}{12}+\frac{5 \mu L^{4}}{2}=0
\end{aligned}
$$

We can write the above relations in a matrix form as follows:

$$
\left(\begin{array}{lll}
b_{11} & b_{12} & b_{13} \\
b_{21} & b_{22} & b_{23} \\
b_{31} & b_{32} & b_{33}
\end{array}\right)\left(\begin{array}{l}
c_{1} \\
c_{2} \\
c_{3}
\end{array}\right)=\left(\begin{array}{c}
\frac{5 L^{4}}{12}-5 \mu L^{2} \\
-\frac{L^{5}}{6}+\frac{10 \mu L^{3}}{3} \\
\frac{L^{6}}{12}-\frac{5 \mu L^{4}}{2}
\end{array}\right)
$$

Substituting the symbolic values for the coefficients given in deflection function:

$$
\bar{U}_{G M}=\frac{5\left(L^{3}-6 \mu L-x^{3}+6 \mu x\right)}{3 A E}
$$

\subsection{Two Parameter Solution}

In this subsection two parameter solutions have been performed in order to assess the effects of residuals of each method. Following relations can be obtained for Sub-domain method:

$$
\begin{aligned}
& \int_{0}^{L / 2} R d x=\int_{0}^{L / 2} E A\left(2 c_{2}(x-L)+c_{1}\right)-10 \mu+5 x^{2} d x=0 \\
& \int_{L / 2}^{L} R d x=\int_{L / 2}^{L} E A\left(2 c_{2}(x-L)+c_{1}\right)-10 \mu+5 x^{2} d x=0
\end{aligned}
$$

$c_{1}$ and $c_{2}$ coefficients can be found from above relations as follows:

$$
\begin{aligned}
& c_{1}=-\frac{5\left(5 L^{2}-12 \mu\right)}{6 A E} \\
& c_{2}=-\frac{5 L}{2 A E}
\end{aligned}
$$

and the axial deflection function is derived:

$$
\bar{U}_{S D M}=\frac{5(L-x)\left(-12 \mu+2 L^{2}+3 L x\right)}{6 A E}
$$


Galerkin method, which minimize the residual, together with boundary conditions, defines the following definite integrals for two terms:

$$
\begin{aligned}
& \int_{0}^{L} A E\left(A E\left(2 c_{2}(x-L)+c_{1}\right)\right. \\
& \left.-10 \mu+5 x^{2}\right)(x-L) d x=0 \\
& \int_{0}^{L} A E 2(x-L)\left(A E\left(2 c_{2}(x-L)-10 \mu+5 x^{2}\right)(x-L)^{2} d x=0\right.
\end{aligned}
$$

The unknown terms can be found as follows:

$$
\begin{aligned}
& c_{1}=-\frac{7 L^{2}-20 \mu}{2 A E} \\
& c_{2}=-\frac{2 L}{A E}
\end{aligned}
$$

The following axial deflection function can be written by using the above equations

$$
\bar{U}_{G M}=\frac{(L-x)\left(-20 \mu+3 L^{2}+4 L x\right)}{2 A E}
$$

In least squares method, following integral equations can be written by using the approximation function:

$$
\begin{aligned}
& \int_{0}^{L} A E\left(A E\left(2 c_{2}(x-L)+c_{1}\right)-10 \mu+5 x^{2}\right) d x=0 \\
& \int_{0}^{L} A E 2(x-L)\left(A E\left(2 c_{2}(x-L)+c_{1}\right)\right.
\end{aligned}
$$

The same relations in Eqs. 47, 48 and 49 are obtained from the results of these integrals. Exact solution can be found as;

$$
\bar{U}_{\text {Exact }}=\frac{5\left(L^{3}-6 \mu L-x^{3}+6 \mu x\right)}{3 A E}
$$

\section{Results and Discussions}

Based on the formulations obtained above with the nonlocal rod model, the axial deflections of nanorod are discussed and investigated here. A computer code is developed based on the different weighted residuals methods. Sufficient number polynomial terms are employed to predict accurate nonlocal axial deflection results in the analysis. 


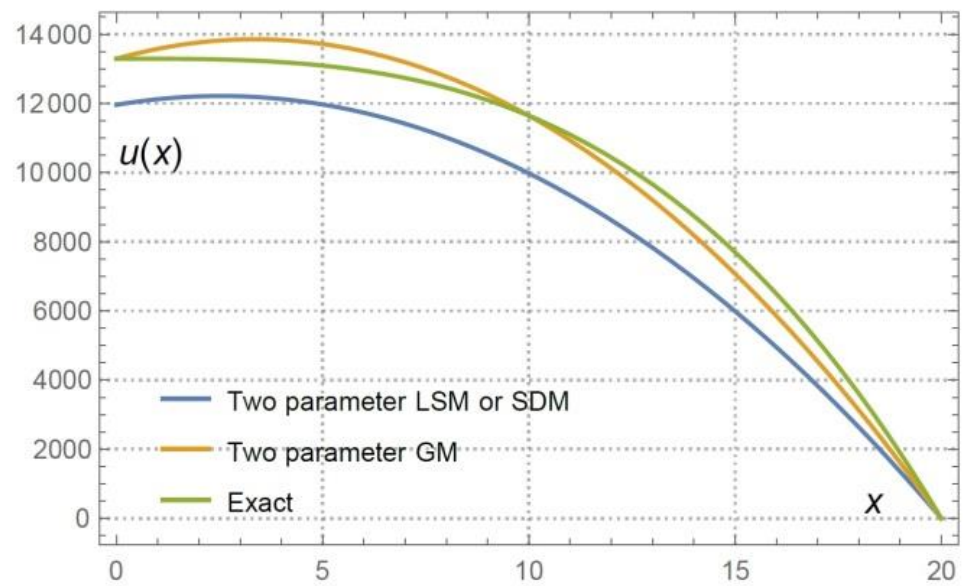

Figure 2. Weighted residuals method with exact solution using two parameters for $L=20 \mathrm{~nm}$

Figure 2 and 3 depict the variation of the axial deflection of nanorods with two different values of lengths. For this study modulus of elasticity is supposed to $1 \mathrm{nN} / \mathrm{nm}^{2}$. Cross sectional area $A=1 \mathrm{~nm}^{2}$, nonlocal parameter $\mu=0.2$ $n m^{2}$ are considered in the analysis.

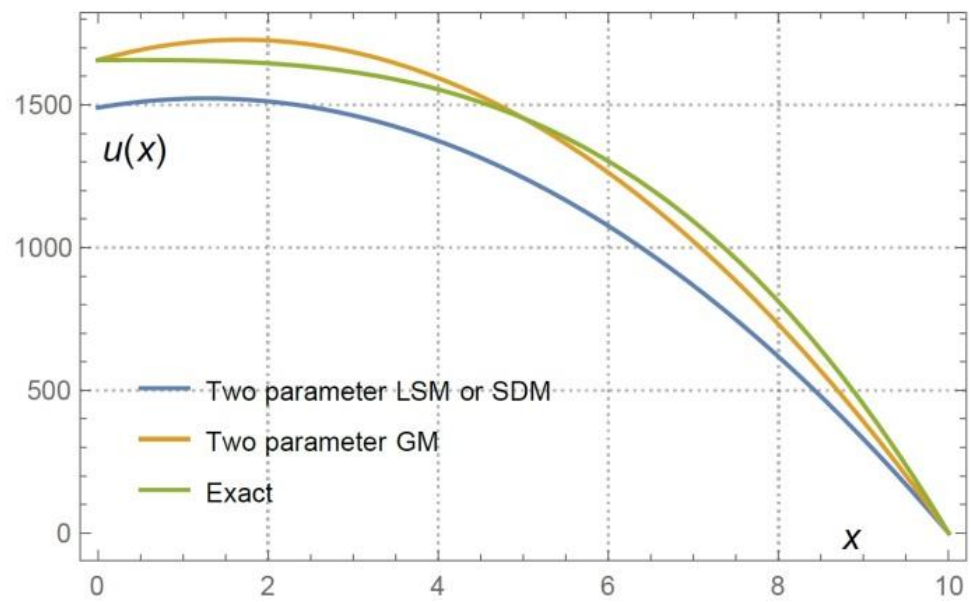

Figure 3. Weighted residuals method with exact solution using two parameters for $L=10 \mathrm{~nm}$

It is apparent from Figures 4 and 5 that, the computed results with minimal effort match closely with that of the exact approach for three terms of polynomials.

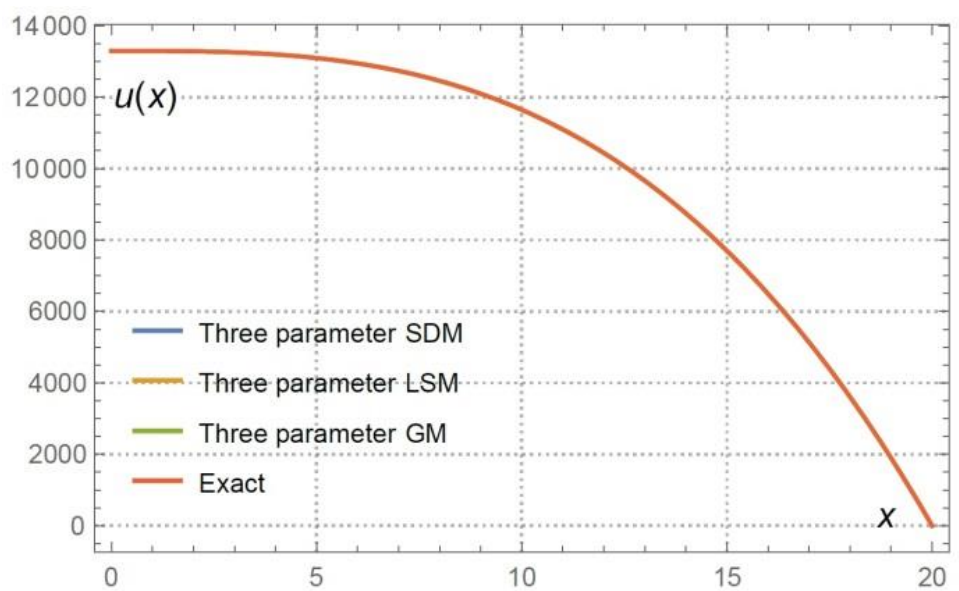

Figure 4. Weighted residuals method with exact solution using three parameters for $L=20 \mathrm{~nm}$ 


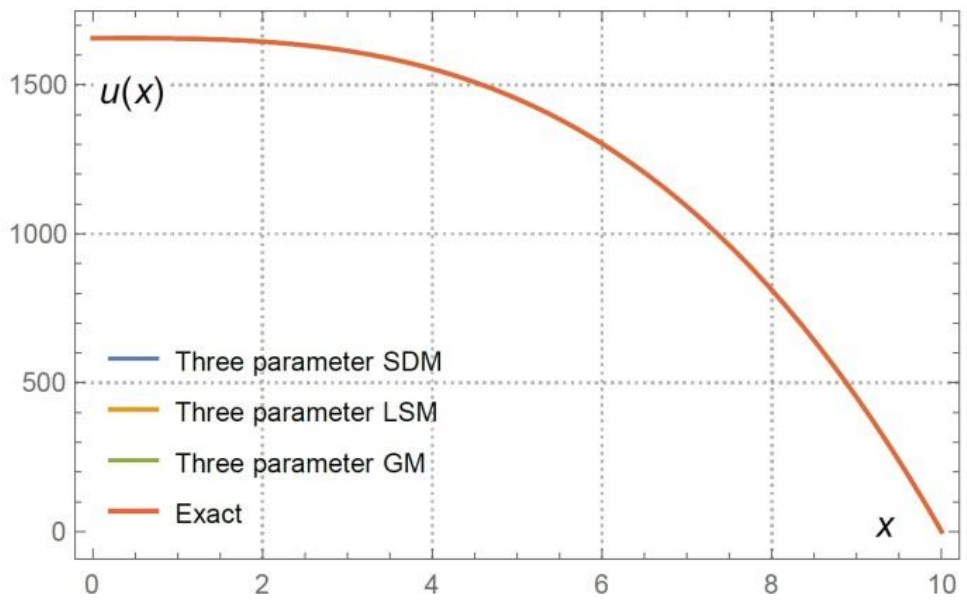

Figure 5. Weighted residuals method with exact solution using three parameters for $L=10 \mathrm{~nm}$

In Figure 6, axial deflections of a nanorod are obtained by applying three different weighted residual methods and the nonlocal parameter are taken as different values. For the second example similar values are used; cross sectional area $A=1 \mathrm{~nm}^{2}$, modulus of elasticity is $1 \mathrm{nN} / \mathrm{nm}^{2}$, nonlocal parameter $\mu=0.2 \mathrm{~nm}^{2}$ are utilized. It can be seen from the Figure 6 that lower elongation amounts are obtained when the non-local elasticity theory is used. The reason for this can be shown as the classical elasticity force boundary condition, which is used controversially in the literature, has never been used.

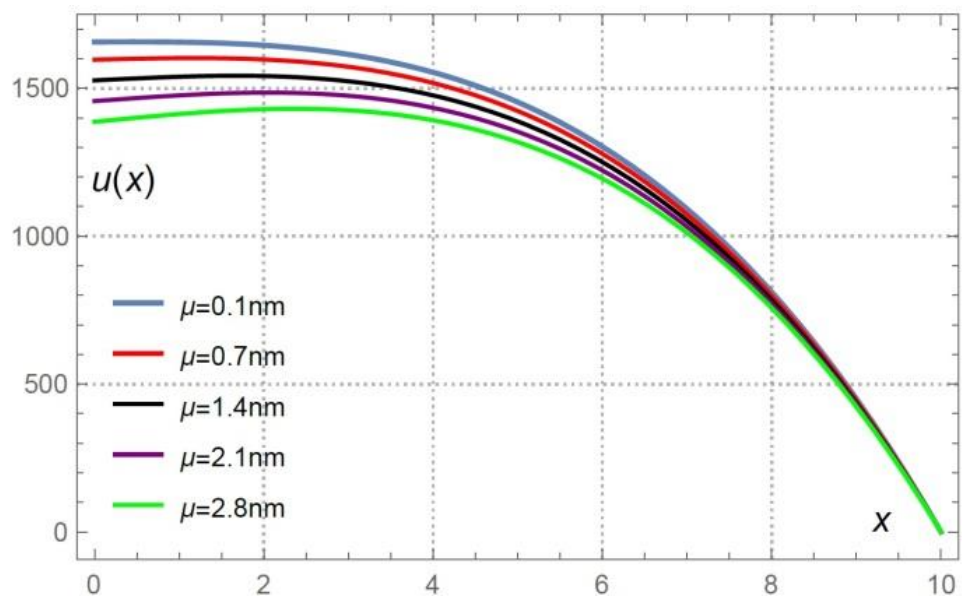

Figure 6. The effect of small scale parameter on the axial deflection for $L=10 \mathrm{~nm}$

The results exhibited certain difference in axial deflection response predictions between nonlocal elasticity and local elasticity, which becomes predominant when the length of nanorod is smaller than $4 \mathrm{~nm}^{2}$. One of the chief contributions of present paper is the derivation of an exact solution using the weighted residuals.

\section{Conclusion}

On the basis of the nonlocal elasticity theory, axial static analysis of nanorods under triangular axial loading conditions is investigated. A polynomial function that defines the axial deflection along the longitudinal axis of the continuum is proposed which satisfied the boundary conditions. The governing ordinary differential equation of the nanorod is solved with the weighted residuals methods (Galerkin, Subdomain, Least squares methods). Numerical values, graphical plots and the influence of the parameters in the polynomials on the axial deflection of the nanorod are presented.

\section{Conflict of Interest}

No conflict of interest was declared by the authors. 


\section{References}

Akbaș, Ș. D. 2019. Longitudinal forced vibration analysis of porous a nanorod Mühendislik Bilimleri ve Tasarım Dergisi, 7(4), 736-743.

Akgöz, B. 2019. Ritz yöntemi ile değișken kesitli kolonların burkulma analizi Mühendislik Bilimleri ve Tasarım Dergisi, 7(2), 452-458.

Akgöz, B., Civalek, Ö., 2013. Buckling analysis of linearly tapered micro-columns based on strain gradient elasticity. Structural Engineering and Mechanics, 48, 195-205.

Akgöz, B., Civalek, Ö., 2015. Bending analysis of FG microbeams resting on Winkler elastic foundation via strain gradient elasticity. Composite Structures, 134, 294-301.

Ansari, R., Gholami, R., Sahmani, S., 2013. Size-dependent vibration of functionally graded curved microbeams based on the modified strain gradient elasticity theory. Arch Appl Mech, 83, 1439-1449.

Arda, M., Aydogdu., M, 2016. Bending of CNTs Under The Partial Uniform Load. Int J Eng Appl Sci, 8, 21-21.

Arda, M., Aydogdu. M., 2017. Longitudinal Vibration of CNTs Viscously Damped in Span. Int J Eng Appl Sci, 9, 22-22.

Arefi, M., Firouzeh, S., Bidgoli, E.M.R., Civalek, Ö. 2020. Analysis of Porous Micro-plates Reinforced with FG-GNPs Based on Reddy plate Theory. Composite Structures, 112391.

Aydoğdu, M., 2009. Axial vibration of the nanorods with the nonlocal continuum rod model. Physica-E Low-dimensional Systems and Nanostructures, 41, 861-864.

Aydogdu, M., Arda, M., 2014. Torsional statics and dynamics of nanotubes embedded in an elastic medium. Compos Struct, 114 80-91.

Aydogdu, M., Arda, M., 2016. Forced vibration of nanorods using nonlocal elasticity. Adv nano Res, 4, 265-279.

Aydogdu, M., Arda, M., Filiz, S., 2018. Vibration of axially functionally graded nano rods and beams with a variable nonlocal parameter. Adv Nano Res, 6, 257-278.

Civalek, Ö., Demir, Ç., 2016. A simple mathematical model of microtubules surrounded by an elastic matrix by nonlocal finite element method. Applied Mathematics and Computation, 289, 335-352.

Civalek, Ö., Uzun, B., Yayll, M.Ö., Akgöz, B. 2020. Size-dependent transverse and longitudinal vibrations of embedded carbon and silica carbide nanotubes by nonlocal finite element method. The European Physical Journal Plus, 135(4), 381.

Dastjerdi, S., Akgöz, B., Civalek, Ö. 2020. On the effect of viscoelasticity on behavior of gyroscopes. International Journal of Engineering Science, 149, 103236.

Demir, C., Civalek, Ö. 2013. Torsional and longitudinal frequency and wave response of microtubules based on the nonlocal continuum and nonlocal discrete models. Applied Mathematical Modelling, 37, 9355-9367.

Ebrahimi, F., Barati, M.R., Civalek, Ö. 2019. Application of Chebyshev-Ritz method for static stability and vibration analysis of nonlocal microstructure-dependent nanostructures. Engineering with Computers, 1-12.

Ece, M.C., Aydogdu, M., 2007. Nonlocal elasticity effect on vibration of in-plane loaded double- walled carbon nano-tubes. Acta Mech., 190, 185-195.

Eringen, A.C., Edelen, D.G.B., 1972. On nonlocal elasticity. International Journal of Engineering Science, 10, 233-248.

Eringen, A.C., 1983. On differential equations of nonlocal elasticity and solutions of screw dislocation and surface-waves. J. Appl. Phys., 54, 4703-4710.

Jalaei, M.H., Civalek, Ö., 2019. A nonlocal strain gradient refined plate theory for dynamic instability of embedded graphene sheet including thermal effects. Composite Structures, 220, 209-220.

Kounadis, A.N., Mallis, J., Sbarounis, A., 2006. Postbuckling analysis of columns resting on an elastic foundation. Arch Appl Mech, $75,395-404$

Li, C., Yao, L.Q., Chen, W.Q., Li, S., 2015. Comments on nonlocal effects in nano-cantilever beams. International Journal of Engineering Science, 87, 47-57.

Li, C. 2014. A nonlocal analytical approach for torsion of cylindrical nanostructures and the existence of higher-order stress and geometric boundaries. Composite Structures, 118, 607-621.

Li, C., Liu, J.J., Cheng, M., Fan, X.L., 2017. Nonlocal vibrations and stabilities in parametric resonance of axially moving viscoelastic piezoelectric nanoplate subjected to thermo-electro-mechanical forces. Composites Part B-Engineering, 116, 153-169.

Liu, J.J., Li, C., Fan, X.L., Tong, L.H., 2017. Transverse free vibration and stability of axially moving nanoplates based on nonlocal elasticity theory. Applied Mathematics and Computation, 45, 65-84.

Reddy, J.N., Pang, S.D., 2008. Nonlocal continuum theories of beam for the analysis of carbon nanotubes. Journal of Applied Physics, 103, 1-16.

Şimşek, M., 2007. Vibration analysis of a single-walled carbon nanotube under action of a moving harmonic load based on nonlocal elasticity theory. Physica-E Low-dimensional Systems and Nanostructures, 43, 182-191.

Thai, S., Thai, H.T., Vo, T.P., Lee, S., 2018. Postbuckling analysis of functionally graded nanoplates based on nonlocal theory and isogeometric analysis. Composite Structures, 201, 13-20.

Uzun, B., Yaylı, M.Ö., 2020a. A solution method for longitudinal vibrations of functionally graded nanorods. International Journal of Engineering and Applied Sciences, 12, 78-87.

Uzun, B., Yayl, M.Ö. 2020b. Nonlocal vibration analysis of Ti-6Al-4V/ZrO2 functionally graded nanobeam on elastic matrix. Arabian Journal of Geosciences, 13(4), 1-10.

Uzun, B., Kafkas, U., Yaylı, M.Ö. 2020a. Axial dynamic analysis of a Bishop nanorod with arbitrary boundary conditions. ZAMMJournal of Applied Mathematics and Mechanics/Zeitschrift für Angewandte Mathematik und Mechanik, e202000039.

Uzun, B., Yaylı, M.Ö., Deliktaş, B. 2020b. Free vibration of FG nanobeam using a finite-element method. Micro \& Nano Letters, 15(1), 35-40

Uzun, B., Civalek, Ö., Yaylı, M.Ö. 2020c. Vibration of FG nano-sized beams embedded in Winkler elastic foundation and with various boundary conditions. Mechanics Based Design of Structures and Machines, 1-20. 
Uzun, B., Kafkas, U., Yaylı, M.Ö. 2020d. Free vibration analysis of nanotube based sensors including rotary inertia based on the Rayleigh beam and modified couple stress theories. Microsystem Technologies, 1-11.

Uzun, B., Kafkas, U., Yaylı, M.Ö. 2020e. Stability analysis of restrained nanotubes placed in electromagnetic field. Microsystem Technologies, 1-12.

Wang, Q., Liew, K.M., 2007. Application of nonlocal continuum mechanics to static analysis of micro and nano-structures. Phys. Lett. A, 363, 236-242.

Yayli, M.Ö., 2014. On the axial vibration of carbon nanotubes with different boundary conditions. IET Micro and Nano Letters, 9, 807-811.

Yayli, M.Ö., Yanik, F., Kandemir, S.Y., 2015. Longitudinal vibration of nanorods embedded in an elastic medium with elastic restraints at both ends. IET Micro and Nano Letters, 10, 641-644.

Yayli, M.Ö., 2016a. Buckling analysis of a microbeam embedded in an elastic medium with deformable boundary conditions. IET Micro and Nano Letters, 11, 741-745.

Yayli, M.Ö., 2016b. A compact analytical method for vibration analysis of single-walled carbon nanotubes with restrained boundary conditions. Journal of Vibration and Control, 22, 2542-2555.

Yayli, M.Ö., 2017. Buckling analysis of a cantilever single-walled carbon nanotube embedded in an elastic medium with an attached spring. IET Micro and Nano Letters, 12, 255-259.

Yoon, J., Ru, C.Q., Mioduchowski, A., 2003. Vibration of an embedded multiwall carbon nanotube. Compos Sci Technol, 63, 15331542. 